\title{
A COMPARATIVE STUDY ON EFFECT OF ORAL ADMINISTRATION OF TURMERIC AND NUTMEG ON MEMORY BOOSTING AND REGAINING IN WISTAR ALBINO RATS
}

\author{
Kumar S.S. ${ }^{1}$, Jissa G. ${ }^{2}$, Setty P.V. ${ }^{3}$, Mukkadan J.K. ${ }^{4}$
}

\begin{abstract}
BACKGROUND AND OBJECTIVES: Although a few drugs are available today for the management of Alzheimer's disease (AD) and many plants and their extracts are extensively employed in animal studies and AD patients, yet no substantial drug or plant extract is able to reverse the AD symptoms adequately. It is tangible that there has been augmenting need for such therapeutic intervention. The present study was undertaken with an objective to compare the memory boosting and regaining effects of oral administration of nutmeg and turmeric which are used commonly as spice in various dishes, as components of teas and soft drinks.
\end{abstract}

MATERIAL AND METHODS: A total of 24 male and female wistar albino rats were used for this study. They were randomly assigned into three groups with 8 rats in each group. The influence of oral intake of nutmeg and turmeric extract on behavioral task performance was studied by using T-maze and radial arm maze and physiological measures relative to a milk control group was investigated.

RESULTS: We have observed significant memory boosting and memory regaining effects of nutmeg when administered orally when compared with control group..However, nutmeg is having strong memory regaining effect than turmeric.

CONCLUSION: We conclude that nutmeg is having strong memory regaining effect than turmeric. However further pharmacological and biochemical investigations will clearly elucidate the mechanism of action and helpful in projecting these plant extracts as a therapeutic target in diabetes research.

KEY WORDS: Memory boosting, Memory retention, Nutmeg extract, Turmeric extract

1. Assistant Professor, Department of Physiology, Travancore Medical College, Kollam, Kerala, India

2. Post Graduate Resident, Department of Physiology, LIMSAR, Angamaly, Kerala, India

3. Assistant Professor, Department of Physiology, Vinayaka Mission's Kirupananda Variyar Medical College \& Hospitals, Salem, Tamil Nadu, India

4. Research Director, Little Flower Medical Research Centre, Angamaly, Kearala, India

\author{
For Correspondence \\ Dr. Sai Sailesh Kumar, \\ Assistant Professor, \\ Department of Physiology, \\ Travancore medical College, Kollam, Kerala, India \\ E-mail: saisailesh.kumar@gmail.com
}




\section{INTRODUCTION}

Learning is acquisition of the information that makes this possible. ${ }^{1}$ Memory is the ability to recall past events at the conscious or subconscious level. ${ }^{2}$ It is the relatively permanent retention and storage form of the learned information. Memory that involves conscious recall of events is called declarative or explicit memory; on the other hand, memory which includes classical conditioning, skills, habits and is largely unconscious, is called Non - declarative or explicit or reflexive memory.

There have been manifold studies to combat this dreadful neurodegenerative disorder for a few decades. Although a few drugs are available today for the management of AD and many plants and their extracts are extensively employed in animal studies and AD patients, yet no substantial drug or plant extract is able to reverse the AD symptoms adequately. It is tangible that there has been augmenting need for such therapeutic intervention. ${ }^{24}$

Medicinal plants improves memory by their acetylcholine esterase inhibitory activity. ${ }^{5}$ Nutmeg, a common household spice, comes from the tree Myristica fragrans, which originates from the Indonesian Banda Islands (also known as the Spice Islands). The Nutmeg plant, Myristica fragrans Houtt, is a member of the small primitive family called Myristicaceae, taxonomically placed between the Annonaceae and Lauraceae. ${ }^{6}$ The name nutmeg comes from Latin, nux muscat, meaning musky nut. Myristicin found in nutmeg has been shown to inhibit an enzyme in the brain that contributes to Alzheimer's disease and is used to improve memory.

Oral administration of nutmeg may have some deleterious effects on the kidneys of adult Wistar rats at higher doses. Nutmeg, the dried seed kernel of Myristica fragrans, MF (Family: Myristicaceae) possesses antifungal, hepatoprotective and antioxidant properties.

Curcumin (Curcuma longa - Haldi) is the source of the spice Turmeric and is used in curries and other spicy dishes from India, Asia and the Middle East. Similar to many other herbal remedies, people first used curcumin as a food and later discovered that it also had impressive medicinal qualities. It has been used extensively in Ayurveda (Indian system of Medicine) for centuries as a pain relieving, anti-inflammatory agent to relieve pain and inflammation in the skin and muscles. It has also proven to have anti-cancer properties. ${ }^{8,9}$ Curcumin holds a high place in Ayurvedic medicine as a "cleanser of the body," and today, science is finding a growing list of diseased conditions that can be healed by the active ingredients of turmeric. ${ }^{10}$ In Ayurvedic practices, turmeric has been used as an anti-inflammatory agent. In a study conducted by the
University of Illinois, researchers discovered that curcumin protected cultured rat cells from damage caused by beta amyloid, a toxin commonly found in the brains of Alzheimer's victims $^{11}$

The present study was undertaken with an objective to compare the memory boosting and regaining effects of oral administration of nutmeg and turmeric which are used commonly as spice in various dishes, as components of teas and soft drinks.

\section{MATERIAL AND METHODS}

Subjects: A total of 24 male and female wistar albino rats were used for this study. They were housed in groups, in propylene cages in an acclimatized $\left(25-27^{\circ} \mathrm{C}\right)$ room and were maintained on a $12 \mathrm{hr}$ light / dark cycle. Food and water was given ad libitum until they aged 30 days at the beginning of the experiment.

\section{Materials:}

T-maze

The T-maze is made of wood with smooth polished surface. It consists of a stem $(35 \times 12 \mathrm{~cm})$, a choice area $(12 \times 12 \mathrm{~cm})$ and two arms $(35 \times 12 \mathrm{~cm})$; at the end of each arm contain a food well. The sidewalls are $40 \mathrm{~cm}$ high. The choice area is separated from the arms by a sliding door.

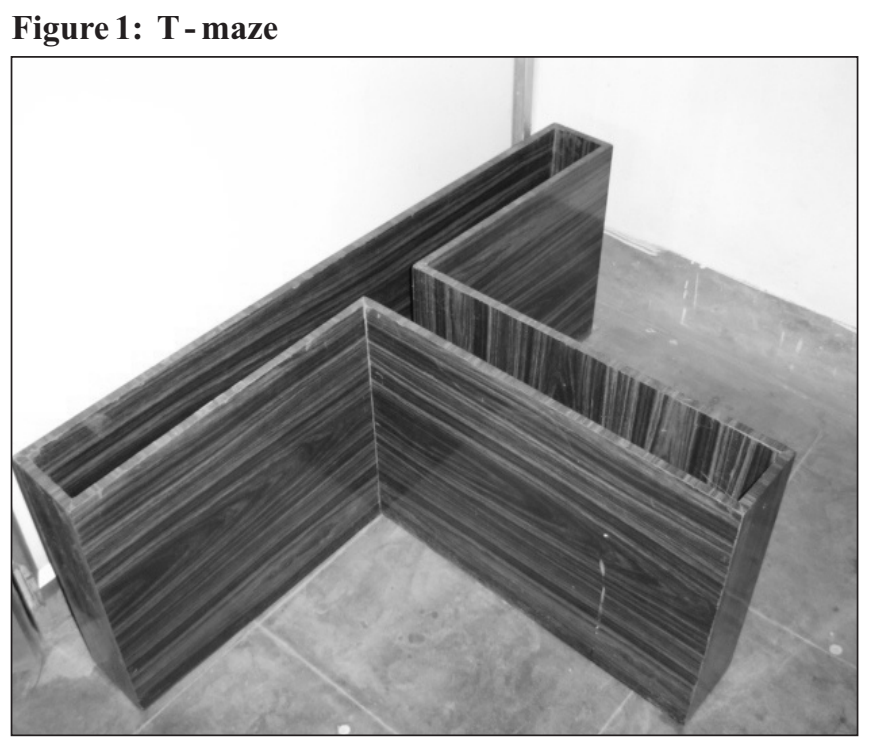

Radial arm maze

Radial arm maze is made of Plexiglass; consist of eight equally spaced arms radiating from an octagonal central platform. Each arm was having a length of $56.2 \mathrm{~cm}$, width of $7.9 \mathrm{~cm}$ and height of $10 \mathrm{~cm}$. The entire maze is elevated $80 \mathrm{~m}$ above the floor for easy locating of spatial cues by rats. 
Figure 2: Radial arm maze

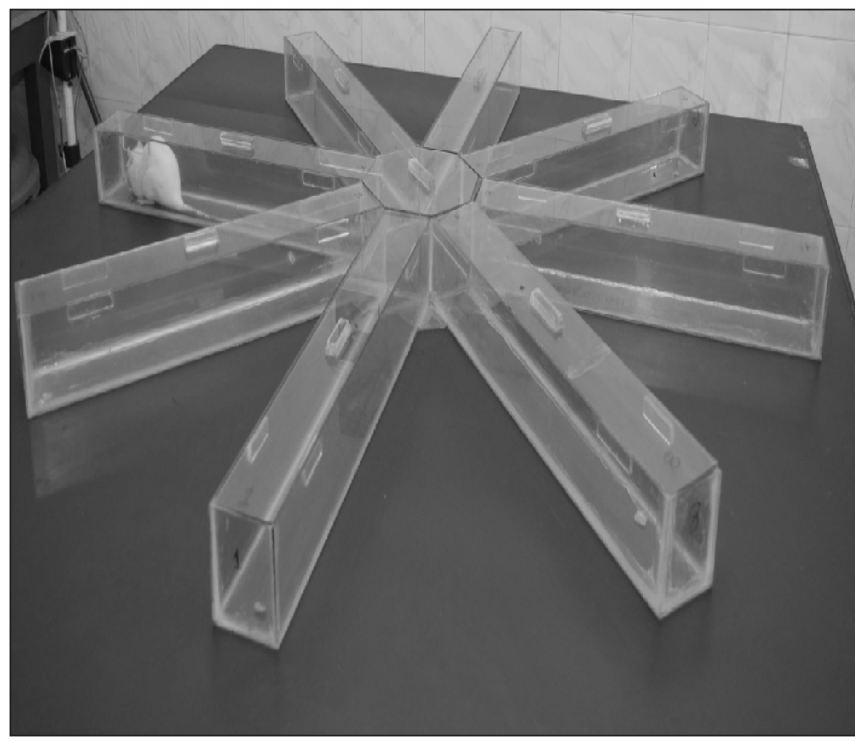

Nutmeg extract

Nutmeg extract is purchased from Kancor ingredients limited, Kancor road, Angamaly, Kerala, India.

\section{Turmeric extract}

Turmeric extract is purchased from Kancor ingredients limited, Kancor road, Angamaly south, India.

\section{Pharmacological drug administration}

Buscopan ${ }^{\circledR}$ tablets manufactured by Cadila Healthcare limited, is used in the present study. Each Buscopan tablet contained Hyoscine (scopolamine) Butylbromide I P $10 \mathrm{mg}$ and excipients (q. s.). The tablets were powdered and mixed with $50 \mathrm{ml}$ sterile $0.9 \% \mathrm{w} / \mathrm{v}$ normal saline. It was administered to the rats as intraperitoneal injection at a dose of $1 \mathrm{mg} / \mathrm{Kg} .{ }^{12}$

Scopolamine was injected at a dose of $1 \mathrm{mg} / \mathrm{Kg}$ body weight of rat, only during the phases where it was assigned. In those groups were the drug is administered, SC was injected 30 minutes before beginning the behavioral trials, every day, either during acquisition or retention depending on the group. ${ }^{13,14,15,16}$

\section{POLYBION}

B complex vitamin manufactured by Merck, Germany is used. It contains thiamine mononitrate $10 \mathrm{mg}$, pyridoxine hydrochloride $3 \mathrm{mg}$, cyanocobalamin $15 \mathrm{mcg}$, riboflavin 10 $\mathrm{mg}$, nicotinamide $100 \mathrm{mg}$, calcium pantothenate $50 \mathrm{mg}$, ascorbic acid $150 \mathrm{mg}$, folic acid $1.5 \mathrm{mg}$, biotin $100 \mathrm{mcg}$.

\section{Experimental design}

They were randomly assigned into three groups with 8 rats in each group.

\section{Control group}

The control rats were given milk for 30 days.

\section{Turmeric group}

The rats in the turmeric group were given $5 \mathrm{mg} / \mathrm{kg}$ body weight of turmeric extract orally for 30 days.

\section{Nutmeg group}

The rats in the nutmeg group were given $12 \mathrm{mg} / \mathrm{kg}$ body weight of nutmeg extract orally for 30 days.

All the rats were fed with pellets and water mixed with B complex tonic liberally in these 30 days. After 30 days, the rats were starved for 48 hours and after 48 hours the behavioral task is performed on T-maze and radial arm-maze for acquisition. This task is continued till we recorded full score without any error.

Now ten days gap was given for the retention of the task. In these ten days only pellets and water mixed with B complex tonic was given to both the groups. On eleventh day behavioral task is performed on T-maze and radial arm-maze and number of trials required to get full score is recorded in both the groups to test memory boosting effect of turmeric and nutmeg. From the next day we have started administration of scopolamine intra peritoneal to both the groups to cause partial amnesia. This procedure continued for 9 days. Scopolamine administration was done at 10 am daily.

Only water mixed B complex suspension is given to both the groups during these 9 days.

From tenth day administration of scopolamine is stopped and turmeric is administered to turmeric group and nutmeg is administered to nutmeg group where milk without turmeric or nutmeg is given to the control group.

This procedure continued for 30 days and food and water mixed with B complex was given to both the groups during these 30 days. On $31^{\text {st }}$ day behavioral task is performed on Tmaze and radial arm maze in both the groups for acquisition and number of trails required to get the full score is recorded. Now ten days gap is given where only food and water mixed with $\mathrm{B}$ complex is given to the rats in both the groups. On eleventh day behavioral tasks were performed on both the mazes to test the retention in both the groups and number of trails required to get the full score is recorded. The memory score was calculated by taking the difference between the number of trials required for acquisition test and number of trials for retention test.

The body weight was maintained at $85 \%$ of the original body weight, throughout experiment. Behavioral experiments were 
conducted in the same room with the same allocentric cues, such as doors, windows, posters and investigator.

\section{T-maze task}

This was analogous to non-matching to sample task, where the rat was rewarded only if the current choice doesn't match the previous one. As reward is used it can also be considered as a learned alternation procedure. In the orientation phase, the starved rats were allowed to spend 10 minutes / day for three days in the T-maze and trained to collect food pellet from the food wells.

During the acquisition test, all the rats were given six trials per day with an inter trial interval of one hour. Each trial consists of four sample and choice run. In the sample run, the rat was placed at the start end of the T-maze stem. The rat was allowed to move towards one arm and collect the food pellet, while keeping the sliding door of other arm closed. In the choice run, the rat was placed at the start end of stem and both arms were kept open.

If the rat visits the same arm as that of sample run, it was recorded as error and the rat was not rewarded with food. Instead, if the rat visits the alternate arm, it was recorded as correct score and the rat was allowed to eat food pellet (reward) in the food well. There was an interval of 30 s between each run. Score was given for alternate selection of arm during choice run and a maximum score of ' 4 ' can be obtained per trial.

\section{Radial arm maze task}

The rats was placed in the centre of the maze and allowed to freely explore the maze for 15 minutes on the first day. The rats were required to take the food pellets from each arm without making a re-entry into the arm already visited. The trial was terminated when the animal takes the food reward from all the eight arms or after 10 minutes if all the eight arms were not visited. Correct sore was give when the visits an arm and collects the food reward, and a maximum score of ' 8 ' can be attained per trial. When a rat reenters an already visited arm it was taken as a working memory error.

\section{Data analysis}

The analysis of data was done by SPSS version 20.0. The Independent- Samples ' $t$ ' test, one-way ANOVA are used for data analysis.

\section{Ethical approval}

The study protocol was approved by Institutional Ethics Committee of Little Flower Medical Research Centre, Angamaly.

\section{RESULTS}

The mean trials of acquisition in control group are $27.83 \pm 2.71$ and in nutmeg group is $16 \pm 2.90$, which indicates that nutmeg group is having memory boosting effect. This is statistically significant $(p<0.001)$. The mean retention of control group is $22.67 \pm 2.42$ and in nutmeg group is $9.67 \pm 2.73$, which indicates that nutmeg group is having memory boosting effect. This is statistically significant $(\mathrm{p}<0.001)$.

The mean trials of acquisition in control group are $27.83 \pm 2.71$ and in turmeric group is $11.33 \pm 1.21$, which indicates that turmeric group is having memory boosting. This is statistically significant $(\mathrm{p}<0.001)$. The mean retention of control group is $22.67 \pm 2.42$ and in turmeric group is $9.17 \pm 1.17$, which indicates that turmeric group is having memory boosting. This is statistically significant $(\mathrm{p}<0.001)$.

The number of mean trials of acquisition in nutmeg group is $16 \pm 2.90$ and in turmeric group is $11.33 \pm 1.21$, which indicates that turmeric group is having more memory boosting effect than nutmeg group. This is statistically significant ( $\mathrm{p}<$ 0.005 ). The mean retention of nutmeg group is $9.67 \pm 2.73$ and in turmeric group is $9.17 \pm 1.17$, which indicates that turmeric group is having more memory boosting effect than nutmeg group. This is not statistically significant $(\mathrm{p}<0.689)$.

The number of mean trials of acquisition in control group is $25.00 \pm 5.22$ and in nutmeg group is $45.00 \pm 3.16$, which indicates that nutmeg group is having memory regaining effect. This is statistically significant $(\mathrm{p}<0.001)$. The mean retention of control group is $17.67 \pm 4.08$ and in nutmeg group is $27.33 \pm 3.08$, which indicates that nutmeg group is having memory regaining effect. This is statistically significant $(\mathrm{p}<0.001)$.

The number of mean trials of acquisition in control group is $25.00 \pm 5.22$ and in turmeric group is $31.50 \pm 1.87$, which indicates that turmeric group is having memory regaining effect. This is statistically significant $(\mathrm{p}<0.017)$. The mean retention of control group is $17.67 \pm 4.08$ and in turmeric group is $17.00 \pm 1.79$, which indicates that turmeric group is having memory regaining effect. This is not statistically significant $(\mathrm{p}<0.722)$.

The number of mean trials of acquisition in nutmeg group is $45.00 \pm 3.16$ and in turmeric group is $31.50 \pm 1.87$, which indicates that turmeric group is having memory regaining effect. This is statistically significant $(\mathrm{p}<0.001)$. The mean retention of nutmeg group is $27.33 \pm 3.08$ and in turmeric group is $17.00 \pm 1.79$, which indicates that turmeric group is having memory regaining effect. This is statistically significant $(p<0.001)$.

The mean trials acquisition in control group is $15.83 \pm 2.64$ and in nutmeg group is $11.67 \pm 2.07$, which indicates that nutmeg group is having memory boosting effect. This is statistically significant $(p<0.012)$. The mean retention of control group is 
$13.00 \pm 2.37$ and in nutmeg group is $8.50 \pm 1.52$, which indicates that nutmeg group is having memory boosting effect. This is statistically significant $(\mathrm{p}<0.003)$.

The mean acquisition of control group is $15.83 \pm 2.64$ and in turmeric group is $13.67 \pm 5.13$, which indicates that turmeric group is having memory boosting effect.

This is not statistically significant $(\mathrm{p}<0.379)$. The mean retention of control group is $13.00 \pm 2.37$ and in turmeric group is $9.33 \pm 4.76$, which indicates that turmeric group is having memory boosting effect. This is not statistically significant $(p<0.122)$.

The mean trials acquisition in nutmeg group is $11.67 \pm 2.07$ and in turmeric group is $13.67 \pm 5.13$, which indicates that nutmeg group is having more memory boosting effect than turmeric group. This is statistically not significant $(p<0.396)$. The mean retention of nutmeg group is $8.50 \pm 1.52$ and in turmeric group is $9.33 \pm 4.76$, which indicates that nutmeg group is having more memory boosting effect than turmeric group. This is not statistically significant $(\mathrm{p}<0.692)$.

The number of mean trials acquisition in control group is $34.33 \pm 3.01$ and in nutmeg group is $32.67 \pm 3.50$, which indicates that nutmeg group is having memory regaining effect. This is statistically significant $(\mathrm{p}<0.398)$. The mean retention of control group is $25.83 \pm 2.79$ and in nutmeg group is $18.50 \pm 2.95$, which indicates that nutmeg group is having memory regaining effect. This is statistically significant $(p<0.001)$.

The number of mean trials of acquisition in control group is $34.33 \pm 3.01$ and in turmeric group is $41.50 \pm 5.68$, which indicates that turmeric group is having memory regaining effect. This is statistically significant $(\mathrm{p}<0.021)$. The mean retention of control group is $25.83 \pm 2.79$ and in turmeric group is $24.83 \pm 5.34$, which indicates that turmeric group is having memory regaining effect. This is not statistically significant $(\mathrm{p}<0.693)$.

The number of mean trials acquisition of nutmeg group is $32.67 \pm 3.50$ and in turmeric group is $41.50 \pm 5.68$, which indicates that nutmeg group is having memory regaining effect. This is statistically significant $(\mathrm{p}<0.009)$. The mean retention of nutmeg group is $18.50 \pm 2.95$ and in turmeric group is $24.83 \pm 5.34$, which indicates that nutmeg group is having memory regaining effect. This is statistically significant $(\mathrm{p}<0.029)$.

Table 1: Anova Memory boosting in Radial arm maze.

\begin{tabular}{|l|l|l|l|l|l|l|}
\hline & & Sum of Squares & df & Mean Square & F & P value \\
\hline \multirow{4}{*}{ Acquisition } & Between Groups & 868.111 & 2 & 434.056 & 75.561 & $<.001$ \\
\cline { 2 - 7 } & Within Groups & 86.167 & 15 & 5.744 & & \\
\cline { 2 - 7 } & Total & 954.278 & 17 & & & \\
\hline \multirow{3}{*}{ Retention } & Between Groups & 703.000 & 2 & 351.500 & 71.735 & $<.001$ \\
\cline { 2 - 8 } & Within Groups & 73.500 & 15 & 4.900 & & \\
\cline { 2 - 7 } & Total & 776.500 & 17 & & & \\
\hline
\end{tabular}

The number of trials for acquisition of these three groups compared by using a one way ANOVA indicates a significant difference with $p<0.001$ between the groups. The number of trials for retention of these three groups compared by using a one way ANOVA indicates a significant difference with $\mathrm{p}<$ 0.001 between the groups.

Table 2: Anova Memory regaining in Radial arm maze

\begin{tabular}{|l|l|l|l|l|c|c|}
\hline & & Sum of Squares & df & Mean Square & F & P value \\
\hline \multirow{4}{*}{ Acquisition } & Between Groups & 1249.000 & 2 & 624.500 & 46.032 & $<.001$ \\
\cline { 2 - 7 } & Within Groups & 203.500 & 15 & 13.567 & & \\
\cline { 2 - 7 } & Total & 1452.500 & 17 & & & \\
\hline \multirow{4}{*}{ Retention } & Between Groups & 401.333 & 2 & 200.667 & 20.523 & $<.001$ \\
\cline { 2 - 7 } & Within Groups & 146.667 & 15 & 9.778 & & \\
\cline { 2 - 7 } & Total & 548.000 & 17 & & & \\
\hline
\end{tabular}

Then the number of trials for acquisition of these three groups compared by using a one way ANOVA indicates a significant difference with $p<0.001$ between the groups. The number of trials for retention of these three groups compared by using a one way ANOVA indicates a significant difference with $\mathrm{p}<$ 0.001 between the groups.

Table 3: Anova Memory boosting in T - maze

\begin{tabular}{|l|l|l|l|l|l|l|}
\hline & & Sum of Squares & df & Mean Square & F & P value \\
\hline \multirow{4}{*}{ Acquisition } & Between Groups & 52.111 & 2 & 26.056 & 2.084 & 0.159 \\
\cline { 2 - 7 } & Within Groups & 187.500 & 15 & 12.500 & & \\
\cline { 2 - 7 } & Total & 239.611 & 17 & & & \\
\hline \multirow{5}{*}{ Retention } & Between Groups & 68.778 & 2 & 34.389 & 3.375 & 0.062 \\
\cline { 2 - 8 } & Within Groups & 152.833 & 15 & 10.189 & & \\
\cline { 2 - 8 } & Total & 221.611 & 17 & & & \\
\hline
\end{tabular}

The number of trials for acquisition of these three groups compared by using a one way ANOVA indicates that the difference between the groups is not significant. The number of trials for retention of these three groups compared by using a one way ANOVA indicates that the difference between the groups is not significant.

Table 4: Anova Memory regaining in $\mathrm{T}$ - maze

\begin{tabular}{|l|l|l|l|l|l|l|}
\hline & & Sum of Squares & df & Mean Square & F & P value \\
\hline \multirow{4}{*}{ Acquisition } & Between Groups & 264.333 & 2 & 132.167 & 7.393 & .006 \\
\cline { 2 - 8 } & Within Groups & 268.167 & 15 & 17.878 & & \\
\cline { 2 - 7 } & Total & 532.500 & 17 & & & \\
\hline \multirow{3}{*}{ Retention } & Between Groups & 189.778 & 2 & 94.889 & 6.321 & .010 \\
\cline { 2 - 7 } & Within Groups & 225.167 & 15 & 15.011 & & \\
\cline { 2 - 7 } & Total & 414.944 & 17 & & & \\
\hline
\end{tabular}

The number of trials for acquisition of these three groups compared by using a one way ANOVA indicates a significant difference with $\mathrm{p}<0.006$ between the groups. The number of trials for retention of these three groups compared by using a one way ANOVA indicates a significant difference with $\mathrm{p}<$ 0.010 between the groups. 


\section{DISCUSSION}

Alzheimer's disease is one of the most common causes of mental deterioration, accounting for around $50-60 \%$ of the overall cases of dementia among persons over 65 years of age. $^{19}$ slowly but surely, Alzheimer's disease (AD) patients lose their memory and their cognitive abilities, and even their personalities may change dramatically. These changes are due to the progressive dysfunction and death of nerve cells that are responsible for the storage and processing of information.

Although drugs can temporarily improve memory, at present there are no treatments that can stop or reverse the inexorable neurodegenerative process. ${ }^{20}$ Although advent of such inhibitors has been effective in function yet there has been augmenting need to quest for new drugs. ${ }^{21}$ There has been growing focus on traditional herbal medicines presently since the failure of existing treatments. ${ }^{22}$ Plants provide wealth of bioactive compounds, which exert a substantial strategy for the treatment of neurological disorders such as Alzheimer's disease. $^{23}$

It was reported that $M$. fragrans significantly decreased acetyl cholinesterase activity as compared with their respective vehicle-treated control groups. Acetyl cholinesterase is an enzyme that inactivates acetylcholine and the central cholinergic pathways play a prominent role in the learning and memory processes. ${ }^{17,18}$ Recent studies on cultured astrocytes obtained from pregnant Sprague-Dawley (SD) rat and neonatal 02-day-old SD rats showed improved neuronal survival by curcumin treatment in NMDA toxicity through the activation of PI3K/MAPK signaling pathways. ${ }^{25}$

Studies employing surface Plasmon resonance experiments, unraveled that the liposome exposing the curcumin derivative (maintaining the planarity) demonstrate considerable affinity for A $\beta 1-42$ fibrils (15 $\mathrm{nM})$, through the exhibition of multivalent interactions. ${ }^{26}$

We have observed significant memory boosting and memory regaining effects of nutmeg and turmeric when administered orally. However, when compared, nutmeg is having strong memory regaining effect than turmeric. This effect may be due to facilitation of acetylcholine activity by decreasing acetyl cholinesterase activity of nutmeg. Hence we recommend further research in this area by investigating compound metabolism to optimize quantification of memory performance following nutmeg consumption.

\section{CONCLUSION}

We conclude that nutmeg is having strong memory regaining effect than turmeric. However further pharmacological and biochemical investigations will clearly elucidate the mechanism of action and helpful in projecting these plant extracts as a therapeutic target in diabetes research

\section{REFERENCES}

1. Ganong WF. Review of medical physiology ( $23^{\text {nd }}$ Ed.) McGraw-Hill Companies. 2005.289

2. Reddy LP. Practical Physiology ( $2^{\text {nd }}$ Ed.). Paras Publicating.289.

3. Jain AK. Text book of physiology. ( $3^{\mathrm{r}}$ Ed. Acichal publishing company. 2007. 1063.

4. Ahuja P. Health benefits of nutmeg.Complete wellbeing.Aug.16, 2009

5. Mukherjee PK, Kumar V, Houghton PJ. Screening of Indian medicinal plants for acetylcholinesterase inhibitory activity. Phytotherapy research. 2007; 21(12): 1142-1145.

6. Joseph J. The Nutmeg, its Botany, Agronomy, Production, Composition and Uses. 1980; 2: 6167

7. Sharma M, Kumar M. Radio protection of swiss albino mice by Myristica fragrans houtt. Journal of radiation research 2007; 48(2): 135-141.

8. Shishodia S, Sethi G, Aggarwal BB. Getting back to roots. Ann NY Acad Sci. 2005;1056: 20617.

9. Ammon HP, Wahl MA. Pharmacology of curcuma longa Planta Med. 1991;57: 17.

10. Youssef KM, El-Sherbeny MA. Synthesis and antitumor activity of some curcumin analogs. Arch Pharm (Weinheim) 2005;338:1819.

11. Park SY, Kim DSHL. Discovery of natural products from Curcuma longa that protect cells from amyloid-beta insult: a drug discovery effort against Alzheimer's disease. J Nat Prod 2002 Sep; 65(9):1227-31.

12. Irena L, Elzbieta D. Scopolamine impairs the response-tochange following observation of the environment but not after its exploration by the rat. Physiol Behav 1985; 34: 625629 .

13. Thouvarecq R, Caston J, Protais P. Cholinergic system, rearing environment and trajectory learning during aging in mice. Physiol Behav.2007; 90: 155164.

14. Harrison FE, Hosseini AH, Dawes SM, Weaver S, May JM Ascorbic acid attenuates scopolamine-induced spatial learning deficits in the water maze. Behav Brain 2009; Res 205: 550558 
15. Robinson L, Harbaran D, Riedel G. Visual acuity in the water maze: sensitivity to muscarinic receptor blockade in rats and mice. Behav Brain Res.2004; 151: 277286.

16. Thouvarecq R, Protais P, Jouen F, Caston J. Influence of cholinergic system on motor learning during aging in mice. Behav Brain Res. 2001; 118: 209218.

17. Dhingra D, Parle M, Kulkarni SK. Comparative brain cholinesterase- inhibiting activity of Glycyrrhiza glabra, Myristica fragrans seeds, and ascorbic acid and metrifonate in mice. Journal of Medicinal Food. Summer 2006, 9(2): 281283.

18. Parle M, Dhingra D, Kulkarni SK. Improvement of mouse memory by myristica fragrans seeds. Journal of Medicinal Food. June2004,7(2):157-161.

19. Francis PT, Palmer AM, Snape M, Wilcock GK. The cholinergic hypothesis of Alzheimer's disease; A review of progress. J Neurol Neurosurg Psychiatry 1999; 66: 137-147.

20. Mattson MP. Pathways towards and away from Alzheimer's disease. Nature. 2004; 430: 631-639.
21. Kim JK, Bae H, Kim MJ, Choi SJ, Cho HY, Hwang HJ et al. Inhibitory effect of poncirus trifoliate on acetyl cholinesterase and attenuating activity against trimethyltin induced learning and memory impairment. Biosci Biotechnol Biochem. 2009; 73: 110512.

22. Drever BD, Anderson WG, Riedel G, Kim DH, Ryu JH, Choi DY, et al. The seed extract of cassia obtusifolia offers neuroprotection to mouse hippocampal cultures. J Pharmacol Sci. 2008; 107:38092.

23. Carpinella MC, Andrione DG, Ruiz G, Palacios SM. Screening for acetylcholinesterase inhibitory activity in plant extracts from Argentina. Phytother Res. 2009; 24: 25963.

24. Obulesu M, Dowlathabad RM. Effects of plant extracts on alzhimer's disease: An insight into theraupeutic avenues. J Neurosci Rural Pract. 2011 Jan-Jun; 2(1): 5661.

25. Lin MS, Hung KS, Chiu WT, Sun YY, Tsai SH, Lin JW et al. Curcumin enhances neuronal survival in N-methyl-Daspartic acid toxicity by inducing RANTES expression in astrocytes via PI-3K and MAPK signaling pathways. Prog Neuropsychopharmacol Biol Psychiatry. 2011.

\section{Journal of Universal College of Medical Sciences is registered in International Standard Serial Number [ISSN 2091-2846 (Print), EISSN 2350-8582 (Online)] and indexed in following:}

\section{Directory of Open Access Journals}

2. Nepal Journals Online

3. Table of Contents

4. Directory of Research Journal Indexing

\section{Ulrichsweb}

6. Asia Journals Online

\section{LibTOC}

8. The GROVE Library

9. NTHRYS

10. Universia

\section{Western Theological Seminary}

